\title{
Gearbox Condition Monitoring Using Advanced Classifiers
}

\author{
P. Večeř, M. Kreidl, R. Šmíd
}

New efficient and reliable methods for gearbox diagnostics are needed in automotive industry because of growing demand for production quality. This paper presents the application of two different classifiers for gearbox diagnostics - Kohonen Neural Networks and the Adaptive-Network-based Fuzzy Interface System (ANFIS). Two different practical applications are presented. In the first application, the tested gearboxes are separated into two classes according to their condition indicators. In the second example, ANFIS is applied to label the tested gearboxes with a Quality Index according to the condition indicators. In both applications, the condition indicators were computed from the vibration of the gearbox housing.

Keywords: Diagnostics, automotive gearbox, Kohonen Neural Network, self-organizing map, ANFIS, classification system, data pre-processing.

\section{Introduction}

A manual automotive gearbox comprises gearwheels, synchronizer pack components, bearings and shafts. Each of these parts is quality checked before assembly. Parts that work together are not paired, because high production capacity lowers the production time. The demand for high-quality completed gearboxes requires the inclusion of an inspection process in the gearbox production line. The inspection process for gearbox diagnostics can be divided into four main parts - signal acquisition, data pre-processing, condition indicator computation and signal classification, as shown in Fig. 1.

Inspection processes are usually based on vibration diagnostics, because the vibration of the gearbox housing during gearbox operation clearly indicates the technical condition of the tested gearbox. An important part of such a diagnostic chain is the classification unit that makes the final decision on the technical condition of the tested object. However, in some cases a classifier can be seen as a further part of the signal processing chain which only simplifies the results from condition computing to a form that is easier for the user to read. An example of this is mapping the test object to easily depicted surfaces.

Neural networks belong to the group of classifiers successfully applied to diagnostics. Neural networks successfully substitute for human power in tasks where the decision is made on the basis of many input features, or when we need to automate the inspection process. Signal conditioning methods often need a trained technician to interpret the results. This is a crucial constraint in present-day industry.

The study of neural networks began in the $19^{\text {th }}$ century, when brain cells were analyzed [4]. The history of artificial neural networks goes back to 1943, when Warren McCulloch and Walter Pitts described how they worked and designed the first simple neural network with electrical circuits [17]. The fundamental concept of how neural nets are learned was introduced in 1949 by Donald Hebb [6]. In 1950, Nathanial Rochester simulated a neural network on IBM electronic calculators [13], which had cells organized into a single layer, and the outputs from these cells were connected to the inputs of other cells. This net was able to meet Hebb's learning rule. In 1958, Frank Rosenblatt created the first Perceptron model [7]. His net learned patterns which occurred regularly and consistently. The first work on neural network classification came from Bernard Widrow and Marcian Hoff, who presented the ADAptive LINear element (ADELINE) [2]. ADELINE was able to recognize a linear pattern. In 1962, Rosenblatt combined his first Perceptron model and ADELINE to create a new Perceptron model [8]. Further progress in neural classification came about in 1972. Two researchers independently came up with the idea of association networks [3]. The first multilayered network [10] appeared in 1975. Then, in 1986, the idea of a multilayer backpropagation network arose [5]. A disadvantage of neural networks is that a trained net cannot be easily visualized. This disadvantage can be overcome by the self-adjusted Fuzzy Interface System known as the Adaptive-Network-based Fuzzy Interface System (ANFIS). ANFIS was introduced by Jung [9] in 1992.

This paper deals with suitable applications of classifiers, based on two different types of approach (SOM and ANFIS) in gearbox quality assessment process. The first part of the paper summarizes the theory of the two networks, while the second part describes experimental results and the third part summarizes the results and their consequences.

\section{Kohonen neural network (Self-Organizing Maps)}

The Kohonen neural network [16] (also called the self-organizing map (SOM)) was presented by T. Kohonen in 1984 . A neuron in SOM is characterized by excitation [12]. Excita-

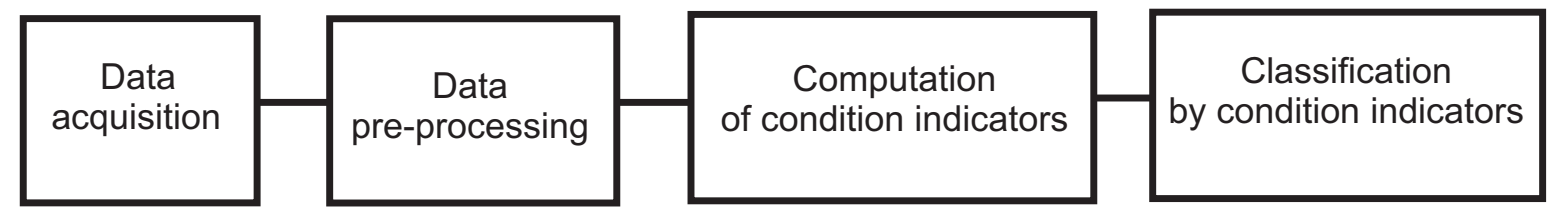

Fig. 1: The inspection process of gearbox diagnostics 


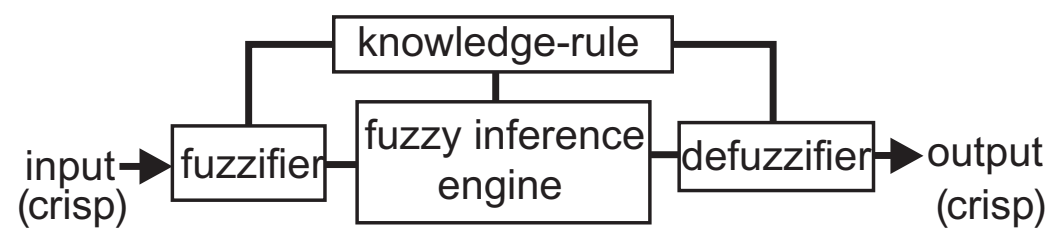

Fig. 2: Basic FIS configuration

tion is usually computed from the Euclidian distance between the weight vector and the input vector. With SOM, a mapping from $n$-dimensional input space into two-dimensional output space can be found. The mapping procedure takes each input vector and compares it with the weight of each neuron. The neuron with the greatest excitation represents a response, and the appropriate input vector is mapped into this location.

\subsection{Topology}

A SOM usually contains only two layers - an input layer and a Kohonen layer. The input layer just provides the distribution of the input vectors to all neurons in the Kohonen layer. The desired mapping from $n$-dimensional input space into two-dimensional space is defined by the Kohonen layer. The neurons in the Kohonen layer are oriented into a rectangular or hexagonal lattice.

\subsection{Using SOM}

In the first stage, called initialization, the weights of all neurons in the SOM have to be set. These weights can be set randomly or can take into account the structure of the input data. After initialization, a new input vector is put into the input layer. The distances $d_{j}$ between the input vector and $j$-th output neuron are computed by equation [12]:

$$
d_{j}=\sum_{i=0}^{N-1}\left[x_{i}(t)-w_{i j}(t)\right]^{2},
$$

where $x_{i} \quad$ is the $i$-th element of input vector $x$ in time $t$,

$w_{i j} \quad$ is the $i$-th value of the weight vector for the $j$-th output neuron in time $t$.

The distance is computed for each output neuron. Then we select the neuron with the shortest distance $d_{w}$, using the equation:

\[ d_{w}=\min _{j}\left(d_{j}\right), \]
where $j=1, \ldots, M$,
$M$ is the total number of neurons in the net.

Finally, the weight vector of the "winning" neuron (the neuron with distance $d_{w}$ ) and the weight of its neighborhood are adjusted.

$$
w_{i j}(t+1)=w_{i j}(t)+\eta(t) \cdot\left[x_{i}(t)-w_{i j}(t)\right],
$$

where $\eta$ is an auxiliary function.

If not all input vectors are put into the input layer, or if the desired number of learning steps is not achieved, this learning process (without initialization) has to be repeated. In the opposite case, the SOM is ready to use.

\subsection{Application of SOM to gearbox diagnostics}

Organizing the input data into meaningful structures is a crucial application of SOM. If the data computed from dif- ferent gearboxes is in the same cluster, we can assume that these gearboxes are in similar technical condition. Unsupervised learning, another important feature of SOM, allows gearbox damage to be identified without the corresponding benchmark vectors.

\section{ANFIS}

Fuzzy expert systems are usually based on a fuzzy inference system (FIS). An important property of fuzzy systems is that they transform human knowledge into the form of fuzzy if-then rules [1]. An FIS is usually composed of four functional units. The basic configuration of FIS is depicted in Fig. 2.

First, the crisp input data is fuzzyfied. Then fuzzy if-then rules are applied. A final decision is obtained from the aggregation process in which all rules are evaluated. Finally, the defuzzification process is applied. Fuzzy inference systems can be divided into three types [9]. For our purposes we will be oriented to type 3, where Takagi and Sugeno's fuzzy if-then rules are used. To design this fuzzy interface system, the parameters of the membership functions and fuzzy if-then rules must be known. However, this is one way to design a fuzzy system without a priori knowledge about membership values and rules. Adaptive Network-based FIS obtains the needed information directly from an input-output data set [9].

\subsection{ANFIS architecture}

The architecture of ANFIS is based on adaptive networks. Adaptive networks are feed-forward networks in which the node parameters are tuned by a learning algorithm to minimize the prescribed error measure. The ANFIS structure for type 3 fuzzy reasoning will be examined in a simple example [9]. Suppose that the knowledge base contains two fuzzy if-then rules (Takagi and Sugeno's type):

Rule 1: If $x$ is $\mathrm{Al}$ and $y$ is $\mathrm{B} 1$, then $\mathrm{fl}=p_{1} x+q_{1} y+r_{1}$ Rule 2: If $x$ is $\mathrm{A} 2$ and $y$ is $\mathrm{B} 2$, then $\mathrm{fl}=p_{2} x+q_{2} y+r_{2}$

The corresponding architecture of ANFIS is illustrated in Fig. 3. Each layer is described below:

Layer 1: The function of this layer is to fuzzify the inputs. A node function can be expressed by the equation:

$$
O_{i}=\mu_{\mathrm{A}_{i}}(x),
$$

where $\mu_{\mathrm{A}}$ is the membership function for linguistic value $\mathrm{A}_{i}$

$x \quad$ is input value.

Layer 2: This layer simply multiplies the incoming membership values according to a node function:

$$
\omega_{i}=\mu_{\mathrm{A}}(x) \times \mu_{\mathrm{B}}(y),
$$

where $\mu_{\mathrm{A}} \quad$ represents the degree to which given $x$ satisfies the quantifier $\mathrm{A}_{i}$, 


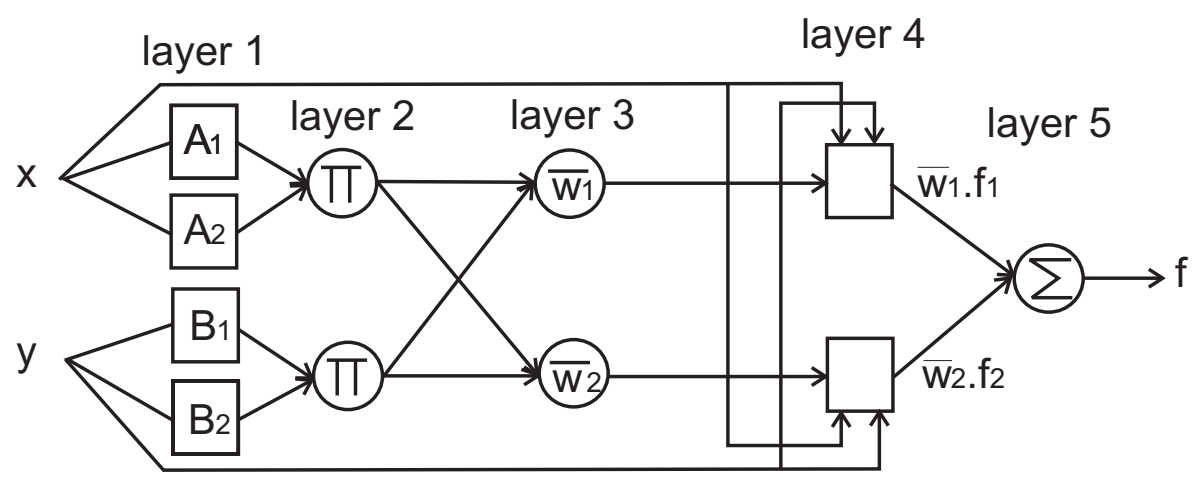

Fig. 3: ANFIS structure

$\mu_{\mathrm{B}} \quad$ represents the degree to which given $y$ satisfies the quantifier $\mathrm{B}_{i}$.

Layer 3: The nodes in this layer calculate the normalized firing strength:

$$
\bar{\omega}_{i}=\frac{\omega_{i}}{\omega_{1}+\omega_{2}}, \quad i=1,2 .
$$

Layer 4: The nodes in this layer compute consequent parts of the fuzzy if-then rules (Takagi and Sugeno's type):

$$
O_{i}=\bar{\omega}_{i} \cdot f_{i}=\bar{\omega}_{i} \cdot\left(p_{i} x+q_{i} y+r_{i}\right) .
$$

Layer 5: In this layer the defuzzification process occurs.

$$
O=\sum_{i} \omega_{i} \cdot f_{i}=\frac{\sum_{i} \omega_{i} \cdot f_{i}}{\sum_{i} \omega_{i}} .
$$

\subsection{Learning algorithm}

The learning algorithm is based on backpropagation and the LMS algorithm [9]. The parameters of the premise membership function are set according to backpropagation. The optimization of the consequent equations is provided via linear least mean squares estimation.

\subsection{Application of ANFIS to gearbox diagnostics}

ANFIS can be used in the same manner as feed-forward neuron networks. The net can classify tested gearboxes into classes defined in advance. In addition to the fact ANFIS is in many cases faster in evaluation and learning, the main advantage is that we can view decision surfaces of the trained net in contrast with the feed forward neuron network [15]. In contrast to SOM, we need to know the benchmark vectors for all classes.

Table 1: Measured gearboxes for 2-class separation using SOM

\section{Experimental results}

To demonstrate a promising method for gearbox fault classifier, two experiments are proposed - one based on a classifier with unsupervised learning - section 4.1 and the other with a classifier that sets its parameter according to supervised learning - section 4.2. The experimental results show only a possible solution of this task. For an adequate evaluation of the features of each classifier, a training dataset with a significantly higher number of tested gearboxes will be needed.

\subsection{Classification with a Self-Organizing Map}

The following experiment was designed to check the performance of the classification system [14]. The vibration data from five gearboxes was classified by the designed inspection system. SOM provides a cluster analysis of the input data. According the technical condition of the tested gearbox, they will be classified into two main clusters ( $G$ and NG). We assume that gearboxes mapped to the same cluster have the same technical condition. The advantage of this classification is that we do not need to know the technical state of each benchmark vector during the learning phase.

\subsubsection{Measured gearboxes}

Five gearboxes were chosen for this experiment. Brief descriptions of the measured gearboxes are given in Table 1.

\subsubsection{Data acquisition}

The vibrations of the gearbox housing were measured during a simulated test drive on the test bench. Two piezoelectric accelerometers were used for vibration signal acquisition. The first transducer was placed near the differential gearing. The second transducer was located near the five-gear gearing. These sensor placements ensured fault signal detection from all gearings. The data from the accelerometers was recorded directly onto the PC hard disk for

\begin{tabular}{|c|l|l|}
\hline & \multicolumn{1}{|c|}{ Class representative } & \multicolumn{1}{c|}{ Technical condition } \\
\hline A & Class G (for $1^{\text {st }}$ and $5^{\text {th }}$ gear) & Working well during all the tests \\
\hline B & Class NG for $1^{\text {st }}$ gear, Class G for $5^{\text {th }}$ gear & $\begin{array}{l}\text { Noisy during the test in first gear. Out of tolerance for dimension on } \\
\text { first gear toothing }\end{array}$ \\
\hline C & Class G for $1^{\text {st }}$ gear, Class NG for $5^{\text {th }}$ gear & Differential out of tolerance because of toothing dimension \\
\hline D & Class G (for $1^{\text {st }}$ and $5^{\text {th }}$ gear) & Bearing on the drive shaft on the tolerance limit \\
\hline
\end{tabular}


off-line analysis by the B\&K multi-analyzer system type 3560 . The B\&K MM0024 photoelectric tachometer probe captured the rotation speed of the drive shaft during the test procedure.

\subsubsection{Data pre-processing and classification}

The raw signal was synchronously averaged and three amplitude features (rms, skewness, and kurtosis) were computed. The computed amplitude features from the first and second transducers were reordered into input vectors. For each gearbox five input vectors were computed. An example of an input vector is shown in Table 2. The SOM size of $4 \times 3$ neurons was chosen and the weights were initialized, using random numbers. A hexagonal lattice type and a Gaussian neighborhood type were selected. After this, the map was trained. The training was done in two phases.

Table 2: Typical values of input vectors for the $1^{\text {st }}$ gear

\begin{tabular}{|l|c|c|c|c|}
\hline & A & B & C & D \\
\hline RMS1 $\left(\mathrm{m} / \mathrm{s}^{2}\right)$ & 4.74 & 5.80 & 1.32 & 2.43 \\
\hline RMS2 $\left(\mathrm{m} / \mathrm{s}^{2}\right)$ & 2.77 & 8.74 & 2.35 & 3.13 \\
\hline Skew1 (-) & 0.35 & 0.31 & -0.09 & -0.17 \\
\hline Skew2 (-) & 0.39 & 0.43 & 0.24 & -0.63 \\
\hline Kur1 (-) & 3.02 & 2.80 & 2.95 & 2.74 \\
\hline Kur2 (-) & 2.16 & 2.19 & 2.56 & 2.99 \\
\hline
\end{tabular}

In the first phase, ten thousand steps were set for training. During the first step the reference vectors of the map were roughly computed. In the second learning phase the reference vectors were fine-tuned. In this step of the procedure the map is learned and can be used for visualizing input vectors.
Sammon's mapping is a nonlinear projection of the multidimensional input vector to a two-dimensional point on a plane, whereby the distances between the image vectors tend to approximate the Euclidean distances of the input vector [12]. The final visualization of the Sammon's mapping after the second learning phase is shown in Fig. 4.

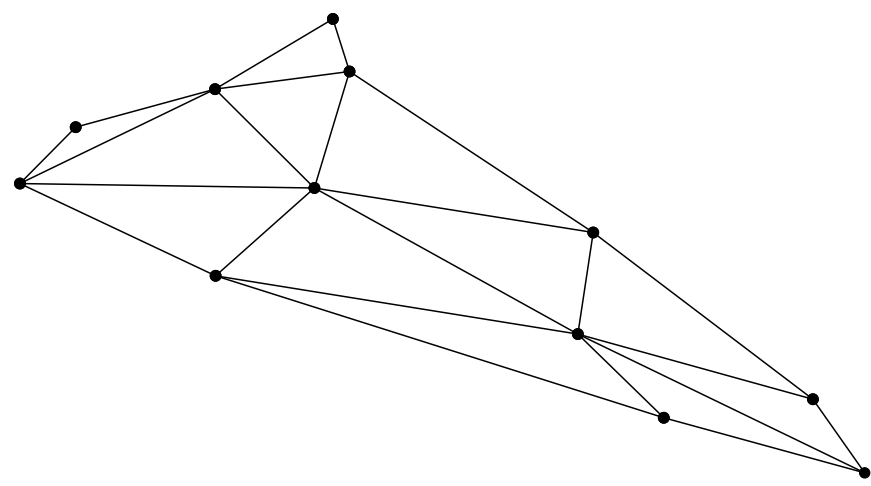

Fig. 4: Sammon's visualization of the learned map after the second phase

The SOM is properly learned. The distances between the reference vector neighboring units and input vector mapping are visualized in Fig. 5. The distances between neurons are depicted using grey scale. Dark grey mean longer distance, light color means a shorter distance. The gearboxes in good technical condition are concentrated on one side. The faulty gearbox is mapped on the right side of the map. These two sides of the map are divided by a sharp border. Thus, SOM divided the tested gearboxes into the two parts of the map: gearboxes in good technical condition are on one side, and the faulty gearbox is on the other side.

The same test was performed for the vibration data retrieved from the second test (five-gears gearing test). The
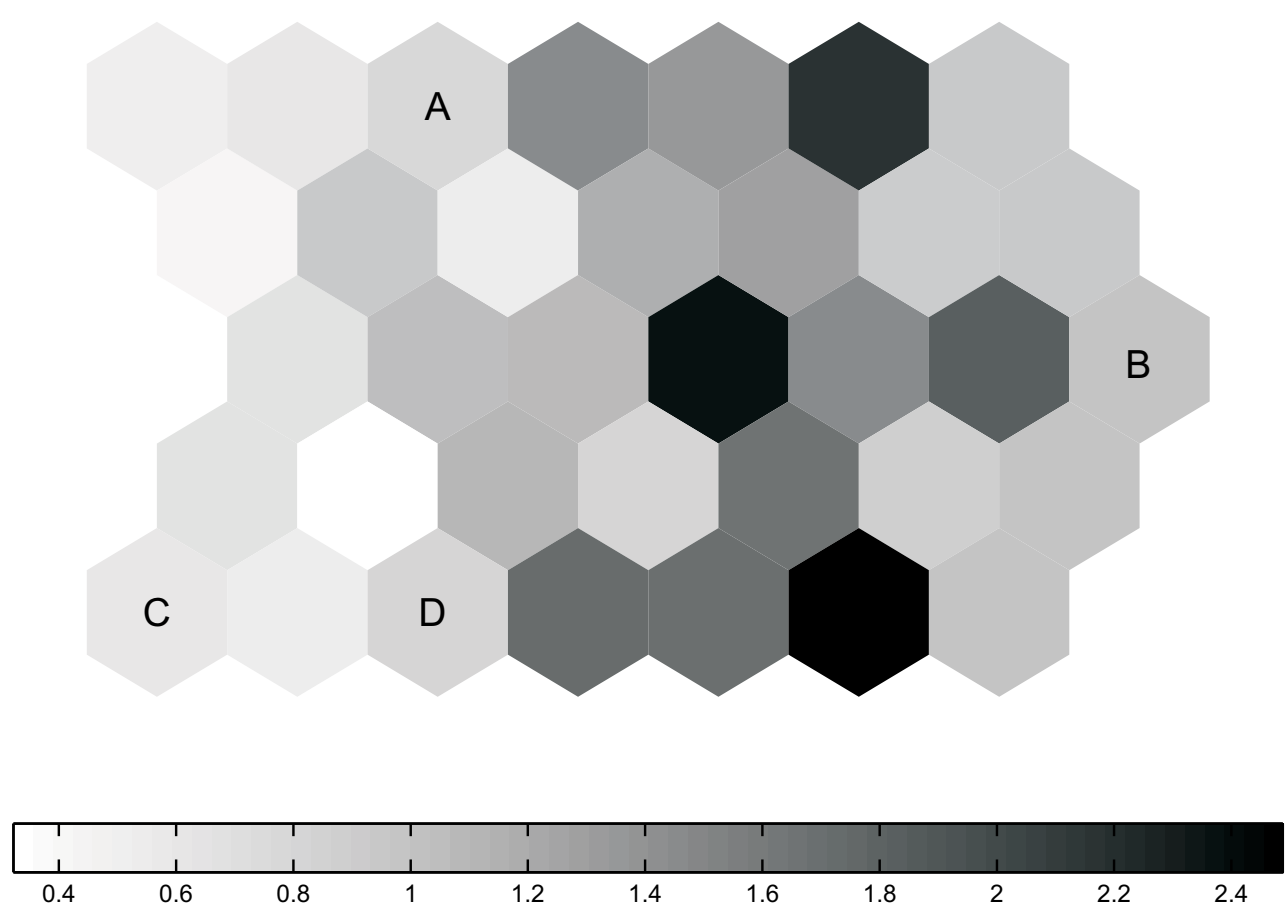

Fig. 5: Distance between reference vectors neighboring neurons and the input vector for first-gear test mapping (lighter gray - shorter distance, darker gray - longer distance) 

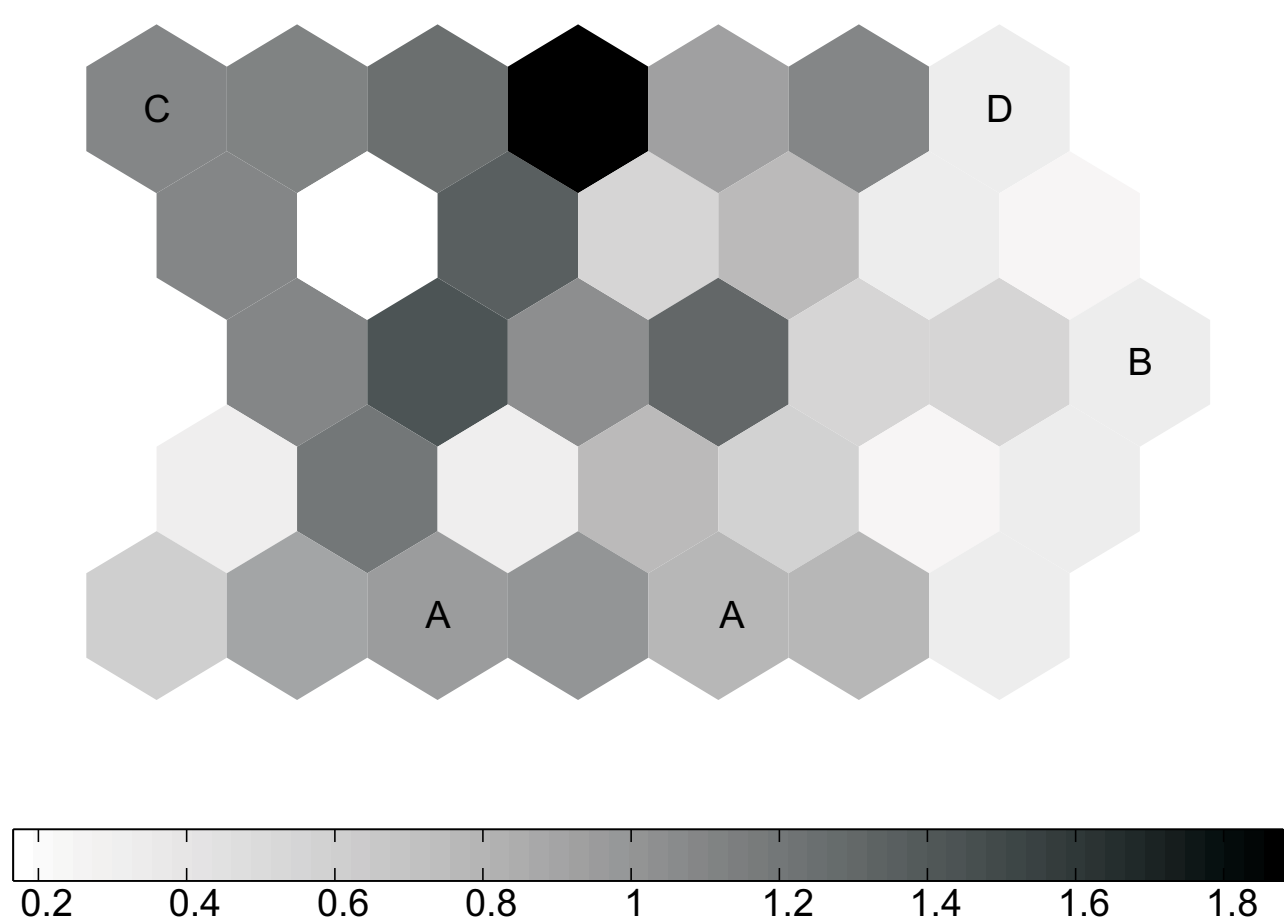

Fig. 6: Distance between reference vectors neighboring neurons and the input vector for five-gear gearing mapping (lighter gray shorter distance, darker gray - longer distance)

distance between the reference vector neighboring units and input vector mapping is visualized in Fig. 6. The gearbox with faulty differential gearing (gearbox $\mathrm{C}$ ) is mapped on the opposite side of the map, distinct from the others. A comparison of the corresponding values in Table 2 shows that the rms value computed from the vibration signal ( $\mathrm{rms} 2$ - second transducer) has a major influence on the vector mapping. The other items have no major effect on the vector mapping. A similar effect was found in the second test.

\subsection{Classification with ANFIS}

The following experiment was designed to check useful features of ANFIS for gearbox diagnostics. The classifier was taught to choose the tested gearboxes with the quality index for each tested gearbox according to a subjective evaluation made by a car tester. As in the previous experiment with SOM, the input data constituted vibration measured on gearbox housings. The data was acquired during a test drive. The quality index classiffied the tested gearboxes into 10 classes (10 - no noticeable noise from gearbox, 0 - unacceptable noise from gearbox). In accordance with the previous example with SOM, we need to have training vectors from the complete quality index scale, if possible. According to the decision surfaces of trained ANFIS, we can decide on the quality of the training data.

\subsubsection{Measured gearboxes}

The subjective quality index for the $3^{\text {rd }}$ gear, deceleration, rated by the test driver, is given in Table 3 .

Table 3: Measured gearboxes for classification with ANFIS

\begin{tabular}{|l|c|c|c|c|c|c|}
\hline Gearbox Label & E & F & G & H & I & J \\
\hline Quality index & 8 & 6 & 7 & $6-5$ & 5 & 8 \\
\hline
\end{tabular}

(C) Czech Technical University Publishing House http://ctn.cvut.cz/ap/

\subsubsection{Data acquisition}

The vibrations of the gearbox housing were measured during a test drive. The test regime was deceleration in $3^{\text {rd }}$ gear. A piezoelectric accelerometer was used for vibration signal acquisition. The vibration transducer was placed on the front side of the gearbox near the switch for the reverse speed. The data from the accelerometer was recorded directly onto the PC hard disk for off-line analysis by the B\&K multi-analyzer system type 3560 . Information on the rotation speed of the gearbox input shaft was acquired from a CAN message send by the Engine Control Unit. The transducer converts a CAN message into an analog impulse signal. An impulse corresponds to one rotation of the input shaft. This signal was connected to the B\&K multi-analyzer.

\subsubsection{Data pre-processing and classification}

Amplitude features (rms, peak, crest factor and kurtosis) were computed from the raw vibration signal. These features were reordered to the input vector and, together with the quality index subjectively evaluated by the car tester; they

Table 4: Typical values of input vectors

\begin{tabular}{|c|c|c|c|c|}
\hline $\begin{array}{c}\text { Tested } \\
\text { Gearbox }\end{array}$ & $\begin{array}{c}\text { RMS } \\
\left(\mathrm{m} / \mathrm{s}^{2}\right)\end{array}$ & $\begin{array}{c}\text { Peak } \\
\left(\mathrm{m} / \mathrm{s}^{2}\right)\end{array}$ & $\begin{array}{c}\text { Crest factor } \\
(-)\end{array}$ & $\begin{array}{c}\text { kurtosis } \\
(-)\end{array}$ \\
\hline E & 45.62 & 515.05 & 11.29 & 6.78 \\
\hline F & 55.67 & 504.09 & 9.05 & 5.76 \\
\hline G & 72.57 & 649.80 & 8.95 & 5.76 \\
\hline H & 49.19 & 441.65 & 8.98 & 6.19 \\
\hline I & 38.67 & 368.66 & 9.53 & 7.25 \\
\hline J & 64.62 & 480.03 & 7.43 & 5.51 \\
\hline
\end{tabular}


were used as training vectors for the net. Typical values of the computed amplitude features for the tested gearboxes are shown in Table 4. The shape of the membership function was chosen as generalized bell-shaped. The two-rule ANFIS was chosen. The decision surfaces for each pair of amplitude features are depicted in Fig. 7. According to the shape of these decision surfaces, it can be decided whether the training data is large enough, and whether the selected amplitude features correspond to the subjective evaluation of the gearbox carried out by the test driver. In an ideal case, the decision surface will have only one peak and the higher the condition indicator values, the lower the quality index will be. The decision surface tuned according to our training data showed clearly that the biggest impact on the quality index value came from amplitude features rms and peak. This figure also shows that in his subjective evaluation the car tester gave a high quality assessment to two gearboxes that had a higher kurtosis value and a low peak value, and vice versa.

The decision surface based on the peak versus crest factor contains two peaks, because the training data does not contain enough samples from gearboxes in variety of technical conditions. The decision surface based on crest factor versus kurtosis should have a lower quality index for a higher kurtosis value. However, as mentioned above, this is only a demonstration of a promising method for a gearbox fault classifier. In order to evaluate the features of the proposed
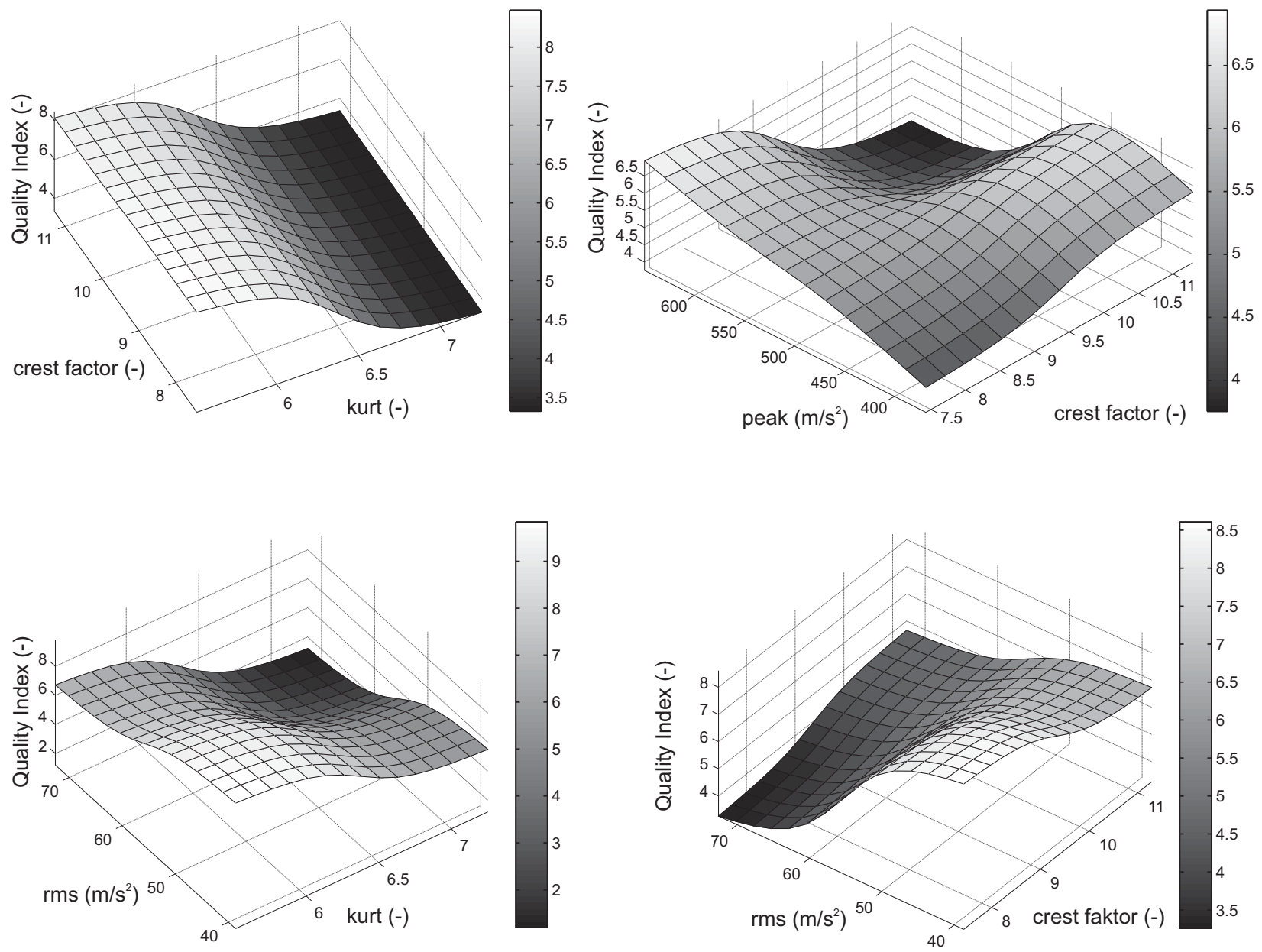

Fig. 7: Decision surfaces of trained ANFIS (2 rules) classifier, a significantly larger training data set containing data from a larger number of tested gearboxes is needed.

If we design the net with more rules, the decision surface will be closer to each subjective evaluation, and the decision surface will be peakier. To avoid these problems, we need more training data that will be equally spread through the whole range of the quality index.

\subsubsection{Conclusion}

The performance of an SOM classifier used for gearbox diagnostics has been demonstrated. One of the major problems of the SOM mapping used in this work is that we cannot be sure how close together or how far apart vectors located on the opposite border of the map are. This problem can be solved by transforming the map into 3-D and projecting it into a round shape (e.g. cylindrical) [11]. However, in our experiment there was a sharp border between the tested gearbox from NG group and other gearboxes. The Kohonen network can correctly classify gearboxes falling into a class, that was not known when the net was trained. We need to know the diagnosis for only one gearbox. The diagnosis for other gearboxes can be evaluated according to their mapping to the benchmark gearbox. Crucial in the application of a Kohonen map is the appropriate size of the map according to the number of test samples. If the map is too large, the tested samples will be too distinct from each other, and vice versa. ANFIS represented a second type of network, which uses supervised learning. A precondition for successful use of this 
type of network is to have training data in advance. The training data must contain enough gearboxes from all classes that we are trying to distinguish between. The advantage of ANFIS is visualizing a decision surface. According to the trained decision surface, we can decide whether our trained data contains enough training samples for the selected net parameters. Our results show that the root mean square value and the kurtosis of the vibration signal have the biggest influence on the final value of the quality index.

\section{Acknowledgments}

This study was supported by research program No. MSM 6840770015 "Research of Methods and Systems for Measurement of Physical Quantities and Measured Data Processing" of the CTU in Prague, sponsored by the Ministry of Education, Youth and Sports of the Czech Republic.

\section{Reference}

[1] Matlab Fuzzy Logic. Mathworks Inc., 2003.

[2] Widrow, B., Hoff, M.: Adaptive Switching Circuits. New York: IRE Wescon Convention Record, 1960.

[3] Olmsted, D. D.: History and Principles of Neural Networks from 1960 to 1990. www.neurocomputing.org, 2006.

[4] Olmsted, D. D.: History and Principles of Neural Networks to 1960. www.neurocomputing.org, 2006.

[5] Rumelhart, D. D., Hinton, G. E., Willliams, R. J.: Learning Representations by Back-Propagating Errors. 1986.

[6] Hebb, D. O.: The Organizing of Behavior. New York: John Wiley \& Sons, 1949.

[7] Rosenblatt, F.: The Perceptron: A Probabilistic Model for Information Storage and Organization in the Brain. Psychological Review, 1958.

[8] Rosenblatt, F.: Principles of Neurodynamics: Perceptrons and the Theory of Brain Mechanisms. Washington D.C.: Spartan books, 1960 .

[9] Jang, J. R.: Neuro FuzzyModeling: Architecture, Analyses and Applications. PhD Thesis, University of California, Berkeley, CA, 1992.
[10] Fukushima, K.: Cognitron: A Self-Organizing Multilayered Neural Network. Biological Cybernetics, 1975.

[11] Jurčík, M.: SOM, 2003.

[12] Šnorek, M.: Neuronové sitě a neuropočítače. Praha: ČVUT, 2002.

[13] Rochester, N., Holland, J. H., Haibt, L. H., Duda, W. L.: Test on a Cell Assembly Theory of the Action of the Brain Using a Large Digital Computer. IRE Transaction of Information Theory IT, 1956.

[14] Večeř, P., Kreidl, M., Šmíd, R.: Application of the Self-organizing Map to Manual Automotive Transmission Diagnostics. In ISSPIT 2003, Darmstadt, Germany, December 2003.

[15] Večeř, P., Kreidl, M., Šmíd, R.: Using Adaptive-Network-based Fuzzy Inference System for the Manual Transmission Diagnostics, 2004.

[16] Kohonen, T.: Correlation Matrix Memories. Berlin: Springer-Verlag, 1984.

[17] McCulloch, W. S., Pitts, W. H.: A Logical Calculus of the Ideas Immanent in Nervous Activity. Bulletin of Mathematical Biophysics, 1943.

Ing. Petr Večeř

e-mail: pvecer@centrum.cz

Doc. Ing. Marcel Kreidl, CSc.

phone: +420224352117

e-mail: kreidl@fel.cvut.cz

Doc. Ing. Radislav Šmíd, Ph.D.

phone: +420224352131

e-mail:smid@fel.cvut.cz

Department of Measurements

Czech Technical University in Prague

Faculty of Electrical Engineering

Technická 2

16627 Prague 6, Czech Republic 